\title{
Fitting a two-component scattering model to polarimetric SAR data
}

\author{
A. Freeman \\ Jet Propulsion Laboratory, Califomia Institute of Technology \\ 4800 Oak Grove Drive, Pasadena, CA 91109 \\ Tel: (818) 3541887 Fax: (818) 3935285 \\ e-mail: tony.freeman $($ jpl.nasa.gov
}

\begin{abstract}
Classification, decomposition and modeling of polarimetric SAR data has received a great deal of attention in the recent literature. The objective behind these efforts is to better understand the scattering mechanisms which give rise to the polarimetric signatures seen in SAR image data.

In this paper, an approach is described, which involves the fit of a combination of two simple scattering mechanisms to polarimetric SAR observations. The mechanisms are canopy scatter from a cloud of randomly oriented oblate spheroids, and a ground scatter term, which can represent double-bounce scatter from a pair of orthogonal surfaces with different dielectric constants or Bragg scatter from a moderately rough surface, seen through a layer of vertically oriented scatterers.
\end{abstract}

An advantage of this model fit approach is that the scattering contributions from the two basic scattering mechanisms can be estimated for clusters of pixels in polarimetric SAR images. The solution involves the estimation of four parameters from four separate equations. The model fit can be applied to polarimetric AIRSAR data at C-, L- and P-Band.

\section{THE MODEL}

The model fit includes two (out of three) simple scattering mechanisms, a situation which is illustrated in Figure 1 for forests. First, for canopy (or volume) scattering, it is assumed that the radar retum is from a cloud of randomly oriented scauterers, exbibiting reflection symmetry. The covariance matrix, which is derived from the scattering matrix by forming cross-products between elements, for scatterens with reflection symmetry is:
$\left(\begin{array}{ccc}S_{m} S_{m}^{*} & \sqrt{2} S_{m} S_{m}^{*} & S_{m} S_{n}^{*} \\ \sqrt{2} S_{m} S_{m}^{*} & 2 S_{m} S_{m}^{*} & \sqrt{2} S_{w} S_{m}^{*} \\ S_{m} S_{m}^{*} & \sqrt{2} S_{w} S_{m}^{*} & S_{w} S_{m}^{*}\end{array}\right)=\left(\begin{array}{ccc}1 & 0 & \rho \\ 0 & \frac{(1-\rho)}{2} & 0 \\ \rho^{*} & 0 & 1\end{array}\right)$

where

$$
0 \leq \rho \leq 1, \arg (\rho)=0
$$

This formulation is less restrictive than an earlier model [1] which had randomly oriented, thin dipoles for the canopy scatter, and is a special case of eq. (1), obtained by setting $\rho$ $=1 / 3$.

The second scattering mechanism is double-bounce scattering, e.g. from a ground-trunk interaction. As in the earlier paper [1], the reflection coefficients for the borizontal and vertical scatterers can be different in this model, as can the propagation delay for $\mathrm{H}$ and $\mathrm{V}$ from radar to scatter and back again. The model for double-bounce scatter is:

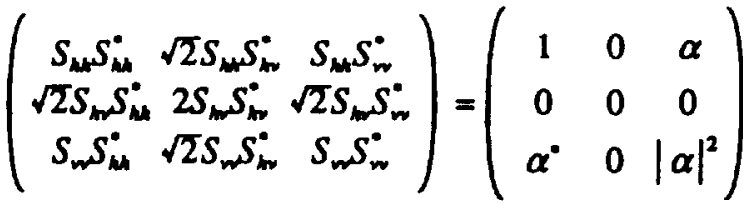

$$
\begin{aligned}
& |\alpha| \leq 1, \arg (\alpha) \approx \pm \pi
\end{aligned}
$$

The third mechanism is direct surface scatter, in which the surface may be tilted in the elevation plane (but not the azimuth for this model), for example a sloping hillside. A phase difference between the $\mathrm{HH}$ and VV backscatter terms is included to model any propagation delay for $\mathrm{H}$ and $\mathrm{V}$ from radar to scatter and beck again , for example by propagation through a canopy layer or a trunk layer. The model for surface scatter is then: 


$$
\begin{aligned}
& \left(\begin{array}{ccc}
S_{m} S_{m}^{*} & \sqrt{2} S_{m} S_{m}^{*} & S_{m w} S_{w}^{*} \\
\sqrt{2} S_{m} S_{m}^{*} & 2 S_{m} S_{m}^{*} & \sqrt{2} S_{m} S_{w}^{*} \\
S_{m} S_{m}^{*} & \sqrt{2} S_{w} S_{m}^{*} & S_{w} S_{w}^{*}
\end{array}\right)=\left(\begin{array}{ccc}
1 & 0 & e^{-2 j} b \\
0 & 0 & 0 \\
e^{-2 j \varphi b} & 0 & |b|^{2}
\end{array}\right) \\
& |b| \geq 1, \arg (b)=0
\end{aligned}
$$

Note this has the same exact form as equation (2), except for the restrictions on the modulus and argument of $\alpha$ and $b$. This is easy to see by setting:

$$
\alpha=e^{-2 j \Downarrow b}
$$

Now consider the situation when only two scattering mechanisms are present, for example, canopy scatter plus double-boumce, or canopy scatter plus direct surface scauler. Assuming that the two scatter components are uncorrelated, and that the like- and cross-polarized returns are uncorrelated, the total second order statistics for the two combined will be the sum of the averaged, measured cross-products for each mechanism. Thus the model for the total backscatter is:

$$
\begin{aligned}
& \left\langle M_{h h} M_{h h}^{*}\right\rangle=f_{c}+f_{g} \\
& \left\langle M_{h v} M_{h v}^{*}\right\rangle=\frac{(1-\rho)}{2} f_{c} \\
& \left\langle M_{v} M_{v v}^{*}\right\rangle=f_{c}+|\alpha|^{2} f_{g} \\
& \left\langle M_{h h} M_{v v}^{*}\right\rangle=\rho f_{c}+\alpha f_{l}
\end{aligned}
$$

where $f_{c}$ and $f_{g}$ are the canopy and ground (double-bounce or direct) scatter contributions to the HH cross section. This model gives four equations in four unknowns (ignoring the cross-products between like-and cross-pol). In general, a solution can be found - whether that solution is consistent with the constraints on the data remains $t 0$ be seen. For example, solutions which yield negative values of $f_{c}$ and $f_{g}$ would not be acceptable.

To solve for $\alpha$, from equation (5a) and (5c), form:

$z_{1}=\left\langle M_{h h} M_{h h}^{*}\right\rangle-\left\langle M_{v} M_{v v}^{*}\right\rangle=f_{s}\left(1-|\alpha|^{2}\right)$

and from equations (5a), (5b) and (5d),

$z_{2}=2\left\langle M_{h v} M_{h v}^{*}\right\rangle+\left\langle M_{h h t} M_{v v}^{*}\right\rangle-\left\langle M_{h h^{\prime}} M_{h h}^{*}\right\rangle=(\alpha-1) f_{t}$

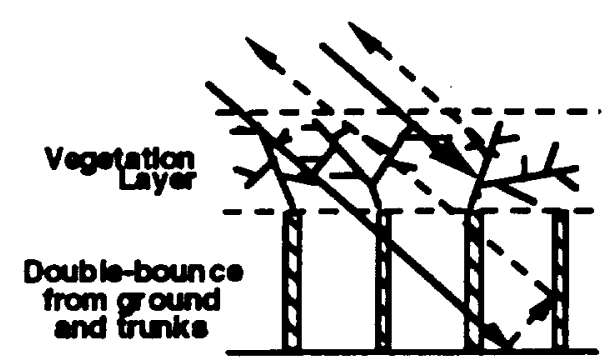

$C$

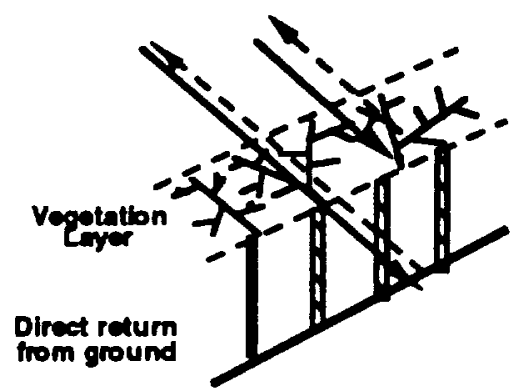

Figure 1: 2-component scattering from forests, showing canopy scatter plus double-bounce (top) and canopy scatter plus single-bounce (bottom)

Then to eliminate $f_{\boldsymbol{g}}$, take the ratio to give:

$$
z_{3}=\frac{z_{2}}{z_{1}}=\frac{(\alpha-1)}{\left(1-|\alpha|^{2}\right)}
$$

which gives:

$$
\left(1-|\alpha|^{2}\right) z_{3}+1-\alpha=0
$$

Taking real and imaginary parts:

$$
\left(1-\left(x^{2}+y^{2}\right)\right) \operatorname{Re}\left(z_{3}\right)+1-x=0
$$

and

$$
\left(1-\left(x^{2}+y^{2}\right)\right) \operatorname{Im}\left(z_{3}\right)-y=0
$$

where 


$$
x=\operatorname{Re}(\alpha), y=\operatorname{Im}(\alpha)
$$

After some algebraic manipulation, it can be seen that:

$$
x=\left(\frac{\operatorname{Re}\left(z_{3}\right) y}{\operatorname{Im}\left(z_{3}\right)}+1\right)
$$

which, on substitution into equation (10b) leads to a quadratic in $y$,

$$
\left(\frac{\operatorname{Re}^{2}\left(z_{3}\right)}{\operatorname{Im}\left(z_{3}\right)}+\operatorname{Im}\left(z_{3}\right)\right) y^{2}+\left(2 \operatorname{Re}\left(z_{3}\right)+1\right) y=0
$$

which has solution:

$$
y=0 \text { or } y=\frac{-\operatorname{Im}\left(z_{3}\right)\left(2 \operatorname{Re}\left(z_{3}\right)+1\right)}{\left|z_{3}\right|^{2}}
$$

This solves for $y$, which is the imaginary part of $\alpha$. The real part of $\alpha(x)$ is found from (11). Once $\alpha$ is known, $f_{c}$ can

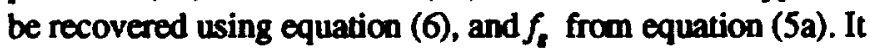
is then straightforward to recover $\rho$ from equation (5b).

Finally, we estimate the contribution of each scattering mechanism to the span, $P$,

$$
\begin{aligned}
& \qquad P=P_{c}+P_{s} \equiv\left(\left|M_{h h}\right|^{2}+2\left|M_{h v}\right|^{2}+\left|M_{v v}\right|^{2}\right) \\
& \text { with } P_{s}=f_{s}\left(1+|\alpha|^{2}\right) \text {, and } P_{c}=f_{c}(3-\rho)
\end{aligned}
$$

$P$ is just $\mathbf{4}$ times the usual expression for total power.

\section{DISCUSSION}

In implementing this approach using AIRSAR data the following anomalous conditions were found:

1. $z_{1}=0$. Causes division by zero in estimating $z_{3}$ using equation (8). Solution adopted was to make $z_{1}$ a very small, positive number.

2. $z_{3}=0$. Causes division by zero in estimating $y$ using equation (13). Solution adopled was to make $\operatorname{Re}\left(z_{3}\right)$ very small, positive number, keeping $\operatorname{Im}\left(z_{3}\right)=0$.
3. Negative values for $f_{c}$. Solution was to average over more pixels, which tended to cure the situation. For one data set, which was initially 4-look, then $4 \times 4$ averaged, a further $3 \times 3$ averaging was needed to eliminate the majority of the negative values. This is a total of 576 data values - the final product is then 120 meter pixels.

Due to speckle, averaging over many pixels was found to be essential for this process to work. Consider the emor in measuring one backscatter $\left(\sigma^{\circ}\right)$ value, which is:

$$
\hat{\sigma}^{\circ}=\sigma^{\circ} \pm \frac{\text { S.D. }\left(\sigma^{\circ}\right)}{\sqrt{N}}
$$

where $\mathrm{N}$ is the number of independent samples used in the estimate. Roughly $67 \%$ of the data values will lie in this (1sigma) range. For the usual speckle distribution for one-look data (exponential distribution), the mean value of $\sigma^{\circ}$ and the standard deviation of $\sigma^{\circ}$ are equal (this is a well-known result). Thus, with averaging:

$$
\hat{\sigma}^{\circ}=\sigma^{\circ}\left(1 \pm \frac{1}{\sqrt{N}}\right)
$$

With $N=64$, the standard emror is still $0.5 \mathrm{~dB}$. Only after averaging 576 samples does the standard enror fall below 0.2 $\mathrm{dB}$. Since one of our assumptions is azimuthal symmetry, the $\mathrm{HH}$ and $\mathrm{HV}$ backscatter should have speckle distributions which are independent of each other. Thus the real part of $z_{2}$, which is estimated using a difference between the HH backscatter and the HV, can have significant errors unless sufficient averaging is carried out. It is also apparent that the HH-HV correlation is not close to zero with only 64 samples averaged together. In fact it only falls below $0.02 \mathrm{~dB}$ after 576 samples have been averaged.

Results of the 2-component fit applied to AIRSAR dea will be presented at the conference.

\section{ACKNOWLEDGMENTS}

The research described in this paper was carried out by the Jet Propulsion Laboratory. California Institute of Technology, under a contract with the National Aeronautics and Space Administration.

\section{REFERENCES}

[1] Freeman, A. and Durden, S., "A Three-Component Scattering Model for Polarimetric SAR Data", EEEE Trans. on Geoscience and Remote Sensing, Vol. 36, No. 3. pp. 963-973, May 1998. 